ARTICLE

https://doi.org/10.1038/s41467-020-19808-4

\title{
SARS-CoV-2 spike-protein D614G mutation increases virion spike density and infectivity
}

Lizhou Zhang1,8, Cody B. Jackson (1) 1,8, Huihui Mou,1, , Amrita Ojha1, Haiyong Peng (1) 1, Brian D. Quinlan', Erumbi S. Rangarajan², Andi Pan', Abigail Vanderheiden 3,4,5,6, Mehul S. Suthar ${ }^{3,4,5,6}$, Wenhui Li (i] ${ }^{7}$,

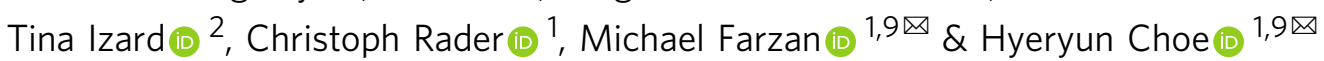

SARS-CoV-2 variants with spike (S)-protein D614G mutations now predominate globally. We therefore compare the properties of the mutated $S$ protein $\left(S^{G 614}\right)$ with the original $\left(S^{D 614}\right)$. We report here pseudoviruses carrying $\mathrm{S}^{\mathrm{G} 614}$ enter ACE2-expressing cells more efficiently than those with SD614. This increased entry correlates with less S1-domain shedding and higher S-protein incorporation into the virion. Similar results are obtained with virus-like particles produced with SARS-CoV-2 M, N, E, and S proteins. However, D614G does not alter S-protein binding to ACE2 or neutralization sensitivity of pseudoviruses. Thus, D614G may increase infectivity by assembling more functional $\mathrm{S}$ protein into the virion.

\footnotetext{
${ }^{1}$ Department of Immunology and Microbiology, The Scripps Research Institute, Jupiter, FL, USA. ${ }^{2}$ Department of Integrative Structural and Computational Biology, The Scripps Research Institute, Jupiter, FL, USA. ${ }^{3}$ Department of Pediatrics, Division of Infectious Disease, Emory University School of Medicine, Atlanta, GA, USA. ${ }^{4}$ Emory Vaccine Center, Emory University School of Medicine, Atlanta, GA, USA. ${ }^{5}$ Yerkes National Primate Research Center, Atlanta, GA, USA. ${ }^{6}$ Emory-UGA Center of Excellence of Influenza Research and Surveillance (CEIRS), Atlanta, GA, USA. ${ }^{7}$ National Institute of Biological Sciences, Tsinghua Institute of Multidisciplinary Biomedical Research, Tsinghua University, Beijing, China. ${ }^{8}$ These authors contributed equally: Lizhou Zhang, Cody B. Jackson, Huihui Mou. ${ }^{9}$ These authors jointly supervised this work: Michael Farzan, Hyeryun Choe. ${ }^{\circledR}$ email: mfarzan@scripps.edu; hchoe@scripps.edu
} 
ntil late 2019, only six coronaviruses were known to infect humans: $\mathrm{HCoV}-229 \mathrm{E}, \mathrm{HCoV}-\mathrm{OC} 43$, SARS-CoV (SARSCoV-1), HCoV-NL63, CoV-HKU1, and MERS-CoV. A seventh human coronavirus, i.e., SARS-CoV-2 detected in late 2019 is closely related to SARS-CoV-1 that emerged in the winter of $2002 / 2003$.

The coronavirus spike $(S)$ protein mediates receptor binding and fusion of the viral and cellular membrane. The $S$ protein extends from the viral membrane and is uniformly distributed as trimers on the virion surface to give the appearance of a crown (corona in Latin). The coronavirus $\mathrm{S}$ protein is divided into two domains: S1 and S2. The S1 domain mediates receptor binding and the S2 mediates downstream membrane fusion ${ }^{1,2}$. The receptor for SARS-CoV-2 is angiotensin-converting enzyme 2 $(\mathrm{ACE} 2)^{3-7}$, a metalloprotease that also serves as the receptor for SARS-CoV- $1^{8}$. A small, independently folded subdomain of S1, described as the receptor-binding domain (RBD), directly binds ACE2 when the virus engages a target cell ${ }^{9-12}$. The S1-S2 junction of SARS-CoV-2 is processed by a furin-like proprotein convertase in the virus-producing cell. In contrast, the S1-S2 junction of SARS-CoV-1 is processed by TMPRSS2 at the cell surface or by lysosomal cathepsins in the target cells ${ }^{13-18}$. The S proteins of both viruses are further processed in the target cell within the S2 domain at the S2' site, an event that is also required for productive infection ${ }^{19,20}$.

Recent analyses of the fine-scale sequence variation of SARSCoV-2 isolates identified several genomic regions of increased genetic variation ${ }^{21-30}$. One of these variations encodes an S-protein mutation, D614G, in the carboxy (C)-terminal region of the S1 domain $^{21-23,26,30}$. This region of the $\mathrm{S} 1$ domain directly associates with S2 (Fig. 1a). The mutant virus with glycine at the residue 614 (G614), replacing aspartic acid (D614), has been shown to rapidly dominate in many locales where it is found ${ }^{21,22}$. Our own analyses of the S-protein sequences available from the GenBank showed a similar result: the G614 genotype was not detected in January and February (among 17 and 33 sequences, respectively) and was observed in a minority of sequences in March $(26 \%$ of 293 sequences), but its frequency increased rapidly by April (65\% of 1511 sequences) and May (70\% of 2544 sequences) (Fig. 1b). This result suggests a potential transmission advantage of G614 virus over D614 virus, although we cannot exclude the contribution of non-stochastic sampling of virus sequences and random founder effects to its current predominance. At least two groups independently noted that D614G mutation correlated with increased viral loads in COVID-19 patients ${ }^{22,31}$, but because this change is also associated with three or four other mutations in other viral proteins including nsp3 and $\mathrm{RdRp}$, the role of the $\mathrm{S}$ protein in these observations remained undefined.

Here we show that the D614G mutation indeed increases pseudovirus (PV) infectivity and that reduced S1 shedding and increased S-protein density in the virion correlate with this increased infectivity.

\section{Results}

The D614G mutation is associated with enhanced infectivity. To determine if the D614G mutation alters the properties of the S protein in a way that could impact transmission or infection, we assessed its role in viral entry. Maloney murine leukemia virus (MLV)-based PVs, expressing enhanced green fluorescent protein (eGFP) and pseudotyped with the $\mathrm{S}$ protein of SARS-CoV-2 (SARS2) carrying the D614 or G614 genotype ( $S^{\mathrm{D} 614}$ and $\mathrm{S}^{\mathrm{G} 614}$, respectively), were produced from transfected HEK293T cells. An $S^{D 614}$ variant, in which the furin-cleavage site between the S1 and $\mathrm{S} 2$ domains is knocked out $\left(\mathrm{S}^{\mathrm{FKO}}\right)$ and generated by replacing the RRAR furin-cleavage motif with SRAS, was also included for comparison. HEK293T cells transduced to express human ACE2 (hACE2-293T) or those transduced with vector alone (Mock293T) were incubated with PVs pseudotyped with S ${ }^{\mathrm{D} 614}$, SG614, or $\mathrm{S}^{\mathrm{FKO}}\left(\mathrm{PV}^{\mathrm{D} 614}, \mathrm{PV}^{\mathrm{G} 614}\right.$, or PV ${ }^{\mathrm{FKO}}$, respectively), and entry levels were assessed $24 \mathrm{~h}$ later. We observed that PVG614 entry into the hACE2-293T cells was markedly higher than PVD614 entry (Fig. 1c, d). To investigate whether this enhanced entry of PVG614 could be affected by TMPRSS2 expression, we conducted similar entry assays in hACE2-293T cells transfected to express hTMPRSS2 (Supplementary Fig. 1c). Entry of PVG614 was similarly enhanced relative to PVD614 in hACE2-293T cells, with or without TMPRSS2 expression (Fig. 1e). Entry of PVFKO was much more efficient than PV D614 (Fig. 1c-e), in contrast to observations from other groups ${ }^{5,12,32}$. This difference is likely explained by the different sequences used to replace the furincleavage motif (Supplementary Fig. 2), and suggests that $S^{F K O}$ used in this study is a better substrate for proteases such as TMPRSS2 and cathepsins that cleave the S1/S2 boundary. Because PVs enter ACE2/TMPRSS2-293T cells via both the endosomal and cell-surface pathways, even in case $\mathrm{PV}^{\mathrm{G} 614}$ is less effective in utilizing TMPRSS2 compared to PV ${ }^{\mathrm{D} 614}$, it may not be detectable. Therefore, we assessed PV entry in a lung epithelial cell line, NCI-H1975, a cell line susceptible to retroviral transduction. Because SARS-CoV-2 target cells in vivo express ACE2 ${ }^{33-35}$, we transduced these cells to express hACE2 (Supplementary Fig. 3b, c) and observed PVG614 entry was again much more efficient than that of PV $\mathrm{P}^{\mathrm{D} 14}$ (Fig. 1f).

The enhanced entry of PVG614 into hACE2-293T and lung epithelial cells is not an artifact of differences in PV production, as the yields of PVs measured by RT-qPCR were similar (Supplementary Figs. 1a, b and 3a). Nor it is the result of differential expression of $S^{D 614}$ and $S^{G 614}$, because the expression of total S protein measured by western blot (WB) in cell lysates and by flow cytometry in transfected cells shows comparable levels for $S^{D 614}$ and $S^{G 614}$ (Supplementary Fig. 4a, b). Together, these data show that $\mathrm{PV}^{\mathrm{G} 614}$ mediates more efficient entry into ACE2-expressing cells than does PV D614.

Superior infectivity of $\mathrm{PV}^{\mathrm{G} 614}$ is associated with decreased S1 shedding and higher S-protein density in the virion. We next investigated the mechanism with which the D614G mutation increased entry of PVG614. Because S1 residue 614 is proximal to the S2 domain, we first investigated whether this mutation contributes to the association between the S1 and S2 domains. To detect both the S1 and S2 domains, we used the S-protein constructs bearing the FLAG tag at both their amino (N)- and Ctermini. PVs pseudotyped with $\mathrm{S}^{\mathrm{D} 614}, \mathrm{~S}^{\mathrm{G} 614}$, and $\mathrm{S}^{\mathrm{FKO}}$ were purified and concentrated by pelleting through a $20 \%$ sucrose layer $^{36}$ and evaluated for their yield and entry efficiency. Yields were similar among PVD614, PVG614, and PVFKO (Fig. 2a), and pelleting the PVs through a sucrose layer did not alter the relative entry efficiency between PVG614 and PV ${ }^{\mathrm{D} 614}$ (Fig. 2b). We analyzed these purified PVs by western blots, detecting the S1 and S2 bands with the anti-FLAG M2 antibody. As shown in Fig. 2c, the most noticeable difference between PVG614 and PV ${ }^{\mathrm{D} 614}$ is markedly higher amounts of the S1 and S2 bands of PVG614 compared to PV ${ }^{\mathrm{D} 614}$. Although Fig. 2a made clear that higher Sprotein density in PV $\mathrm{PV}^{\mathrm{G} 14}$ was not a consequence of higher PV yield, we also confirmed this point by detecting the retroviral protein p30 by western blot (Fig. 2c). The densities of p30 bands were comparable in all PVs, indicating that the higher S-protein incorporation observed with $\mathrm{PV}^{\mathrm{G} 614}$ was due to the different incorporation efficiencies between $\mathrm{S}^{\mathrm{D} 614}$ and $\mathrm{S}^{\mathrm{G} 614}$. Indeed, densitometric analyses showed there was 4.7 times more S1 + S2 band in PVG614 compared to PVD614 (Fig. 2d). We also observed 
a
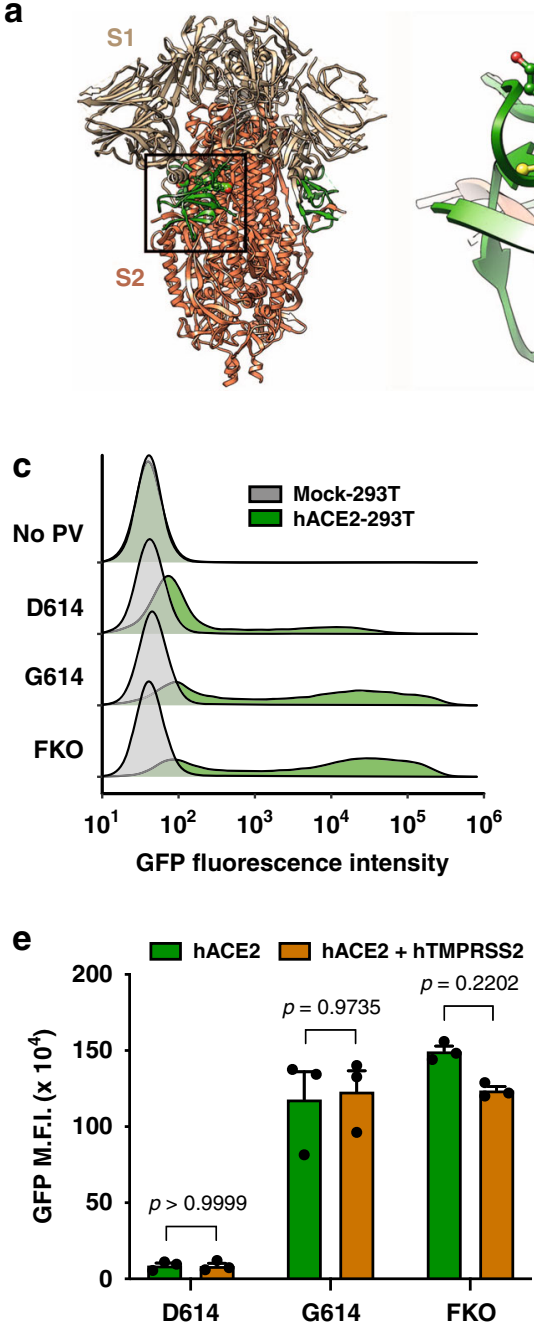

b

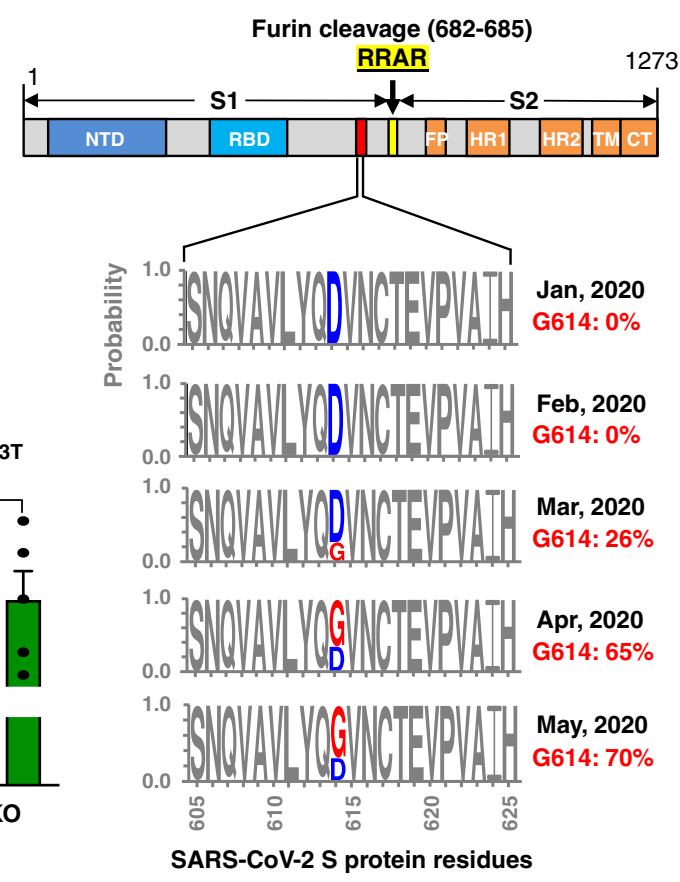

\section{3}

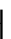

f

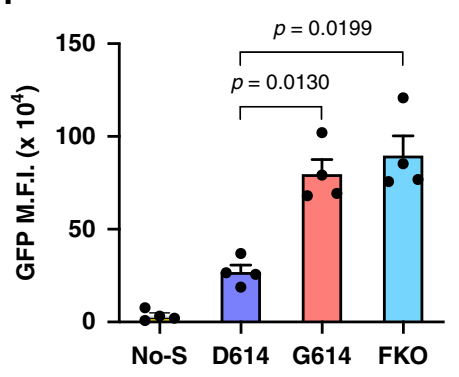



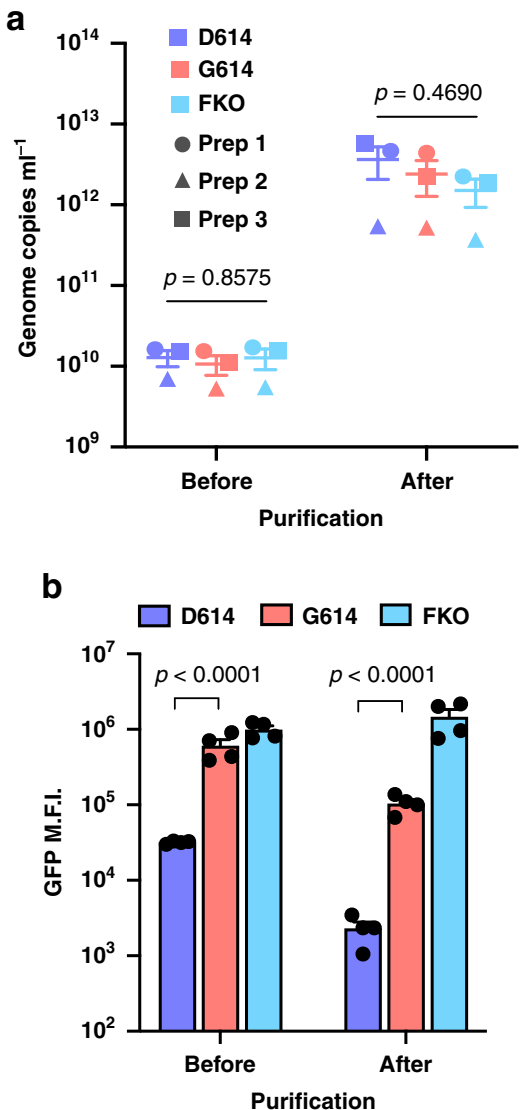

C
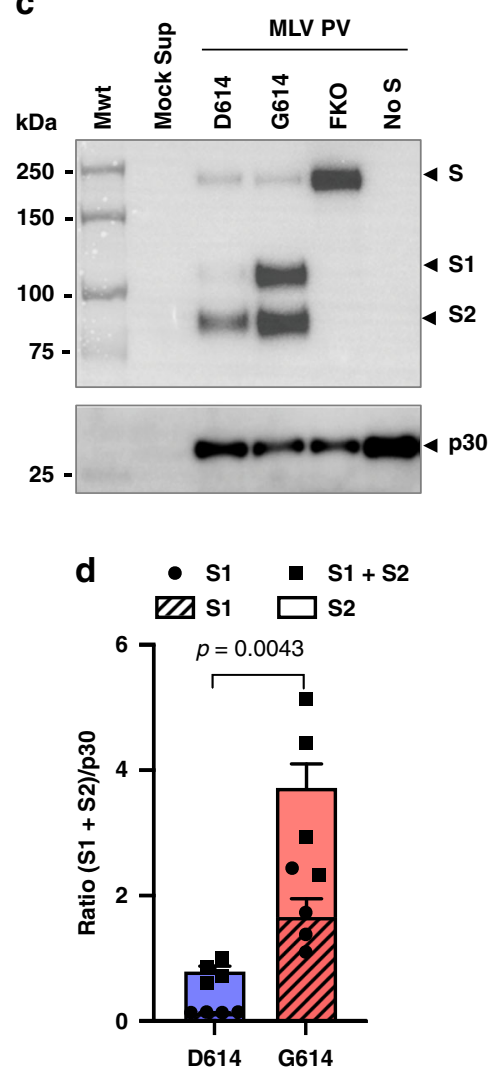

e

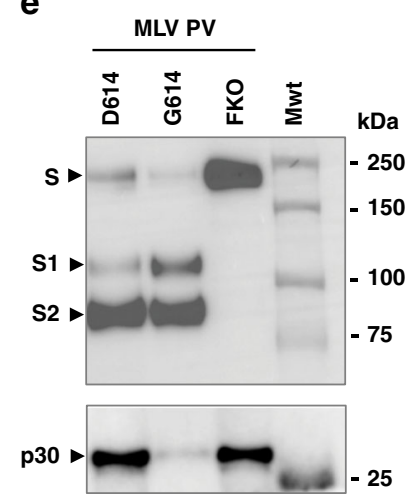

$\mathbf{f}$

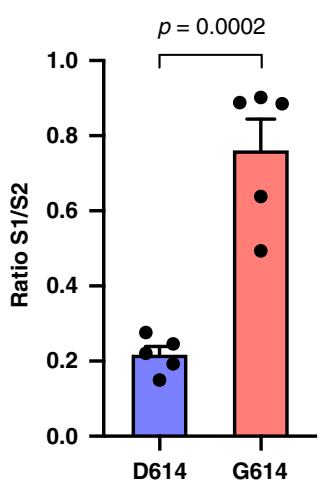

Fig. 2 Superior infectivity of PVG614 is associated with decreased S1 shedding and higher level of S protein in the virion. a-f Indicated MLV PVs produced with the S protein containing the FLAG tag at both the $\mathrm{N}$ - and C-termini were purified by pelleting through a $20 \%$ sucrose layer. PV yields were assessed by RT-qPCR (a). The same symbols in different PV groups indicate they are from the same batch. The same PVs were assessed for their infectivity in Mock and hACE2-293T cells (b). Mean \pm SEM of $n=3$ biologically independent PV batches (a) and $n=4$ experiments using those three PV batches $(\mathbf{b})$ are shown. The same amount $\left(1 \times 10^{10} \mathrm{vg}\right.$ per lane) (c, d) or different amounts to more accurately compare the S1 and S2 ratio $(\mathbf{e}, \mathbf{f})$ of the purified PVs were analyzed by WB (western blot) using the anti-FLAG M2 antibody or anti-p30 MLV gag antibody. The same PVs visualized by silver stain are shown in Supplementary Fig. 5. Total virion S protein (d) and the S1:S2 ratio (f) of PVD614 and PVG614 were calculated from $n=4$ (d) or $n=5$ (f) WBs performed with three independently prepared PV batches and presented as mean \pm SEM. The $p$ values by one-way ANOVA (a), two-way ANOVA with Sidak multiple comparison tests of log-transformed data (b), or two-sided unpaired Student's $t$-test (d, f) are indicated. GFP green fluorescent protein, MLV Maloney murine leukemia virus, PV pseudovirus, FKO furin-cleavage knockout mutant, M.F.I. mean fluorescence intensity.

particles (VLPs) composed only of native SARS-CoV-2 proteins, specifically nucleoprotein $(\mathrm{N})$, membrane protein $(\mathrm{M})$, envelope protein (E), and S protein ${ }^{37}$. VLPs were purified and analyzed in the same way as MLV PVs. S-protein bands were detected with the anti-FLAG M2 antibody, and N protein with pooled plasma derived from convalescent COVID-19 patients. As with PVs, the $\mathrm{S} 1: \mathrm{S} 2$ ratio and total $\mathrm{S}$ protein on the virion were again much higher in the VLPs carrying $\mathrm{S}^{\mathrm{G} 614}$ (VLPG614) compared to those carrying $S^{D 614}$ (VLPD614) (Fig. 3d-f). Thus, enhanced entry of PV containing the D614G mutation correlates with reduced shedding of the S1 domain and greater incorporation of functional spikes into the virion. To exclude an artifact associated with S-protein tags, we similarly characterized untagged S proteins (Supplementary Fig. 7). Again greater virion incorporation of $S$ protein and higher ratios of $\mathrm{S} 1$ to $\mathrm{S} 2$ were observed with $\mathrm{PV}^{\mathrm{G} 614}$ compared with PVD614.

We next asked if higher stability of PV $\mathrm{PV}^{\mathrm{G} 614}$ spikes correlated with slower loss of PV infectivity over time. PVs left at room temperature for up to $48 \mathrm{~h}$ were used in hACE2-293T entry assays. Under these conditions, we observed no difference in the rate of inactivation among $\mathrm{PV}^{\mathrm{D} 614}, \mathrm{PV}^{\mathrm{G} 614}$, or PV ${ }^{\mathrm{FKO}}$ (Supplementary Fig. 8a), indicating that loss of infectivity by these PVs is attributed to factors other than differences in their $S$ proteins.
This unexpected result suggested the possibility that most S1domain shedding from $\mathrm{S}^{\mathrm{D} 614}$ occurs before the test period. To evaluate this possibility, we purified PVs from intracellular compartments and observed that S1 shedding had already taken place (Supplementary Fig. 8b). These data show that low stability of $\mathrm{S}^{\mathrm{D} 614}$ did not accelerate PV $\mathrm{V}^{\mathrm{D} 614}$ inactivation, because most S1 shedding occurred prior to PV release from the cell.

The D614G mutation neither increases $S$ protein affinity for ACE2 nor makes PV more resistant to neutralization. It has previously been speculated that D614G mutation promotes an open configuration of the $S$ protein ${ }^{22,23}$ that is more favorable to ACE2 association ${ }^{5,38}$. To explore this possibility, we investigated whether ACE2 binding to $S^{G 614}$-spikes, S-protein trimers, was more efficient than to $S^{\text {D614 }}$-spikes. HEK293T cells transfected to express each of these $S$ proteins were assessed for their binding of hACE2 immunoadhesin, whose enzymatic activity was abolished by mutation (hACE2-NN-Ig). We previously showed hACE2NN-Ig bound SARS-CoV-1 RBD with an equivalent affinity as hACE2- $\operatorname{Ig}^{39}$. The $\mathrm{S}$ proteins used in this study are fused to the FLAG tag at the C-terminus, but not at the $\mathrm{N}$-terminus, thus allowing for the measurement of total $\mathrm{S}$ protein expression with 
a

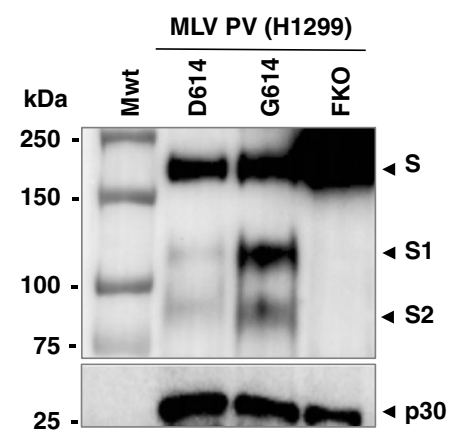

d

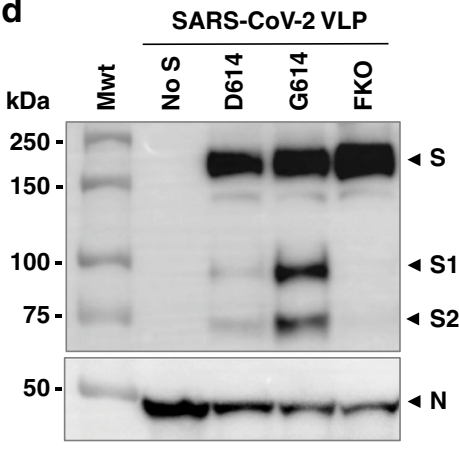

b

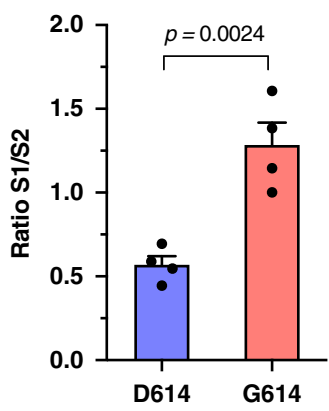

e

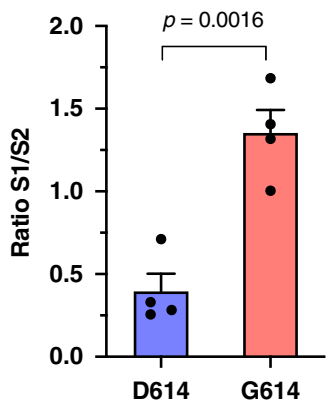

C

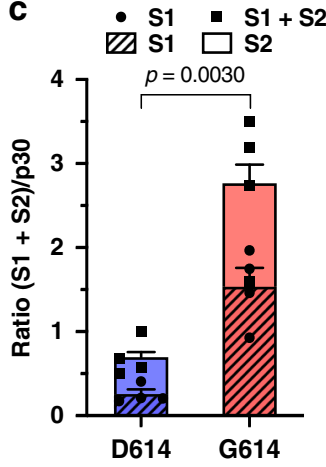

f

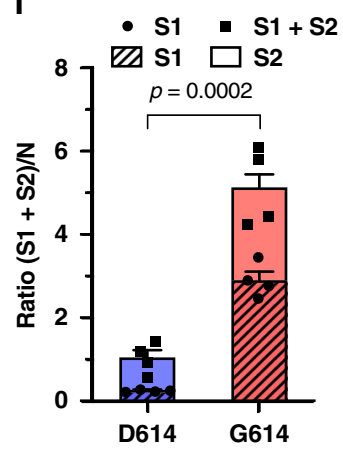

Fig. 3 SARS-CoV-2 VLPG614 and lung epithelial cell-produced PVG614 also exhibit decreased S1 shedding and increased total virion S protein. a-f PVS produced from NCI-H1299, a lung epithelial cell line (a-c), and VLPs (virus-like particles) produced from HEK293T cells by transfecting M, N, E, and S proteins of SARS-CoV-2 (d-f) are analyzed. The S protein is FLAG tagged at both the N- and C-termini. PVs were harvested at $48 \mathrm{~h}$ and VLPs at $43 \mathrm{~h}$ and purified by pelleting through a $20 \%$ sucrose layer. The $\mathrm{S}$ protein bands were visualized using the anti-FLAG tag M2 antibody (a, d), p30 band with an antip30 antibody (a), and the $\mathrm{N}$ protein band using pooled convalescent plasma (d). Representative images of WBs (western blot) performed with $n=4$ independently prepared PV (a) or VLP (d) batches are shown. The S1:S2 ratio and the difference in total virion $\mathrm{S}$ protein incorporation are presented as mean \pm SEM, which were calculated from those four PV WBs $(\mathbf{b}, \mathbf{c})$ or four VLP WBs $(\mathbf{e}, \mathbf{f})$. The $p$ values by two-sided unpaired Student's $t$-test are indicated $(\mathbf{b}, \mathbf{c}, \mathbf{e}, \mathbf{f})$. MLV Maloney murine leukemia virus, PV pseudovirus, FKO furin-cleavage knockout mutant.

an anti-FLAG antibody. As Supplementary Fig. 9a shows, total expression of $\mathrm{S}^{\mathrm{D} 614}, \mathrm{~S}^{\mathrm{G} 614}$, and $\mathrm{S}^{\mathrm{FKO}}$, assessed by FLAG-tag staining of permeabilized cells, was comparable. However, hACE2-NN-Ig binding to cells expressing $\mathrm{S}^{\mathrm{G} 614}$ - and $\mathrm{S}^{\mathrm{FKO}}$-spikes was substantially higher than that to cells expressing $S^{D 614}$-spikes (Fig. 4a and Supplementary Fig. 9a). There are several explanations for this observation. First, $S^{G 614}$ increased ACE2 association by promoting an open configuration of the RBD that was suggested more favorable to ACE2 binding 5,22,23,38. Second, this mutation actually increased S1 affinity to ACE2, although the residue 614 was outside of the RBD. Third, SG614 increased total ACE2 binding by reducing S1-domain shedding. To differentiate these possibilities, we appended the Myc-tag to the N-terminus of the S-proteins that are FLAG tagged at its C-terminus and repeated the experiment, this time detecting the $\mathrm{S} 1$ domain using an anti-Myc antibody. As Supplementary Fig. 9b shows, while total expression of $\mathrm{S}^{\mathrm{D} 614}, \mathrm{~S}^{\mathrm{G} 614}$, and $\mathrm{S}^{\mathrm{FKO}}$, assessed by FLAG-tag staining of permeabilized cells, is similar, the levels of $S^{\mathrm{G} 614}$ - and $\mathrm{S}^{\mathrm{FKO}}$-spikes on the cell surface, measured by an anti-Myc antibody, were considerably higher than that of $\mathrm{S}^{\mathrm{D} 614}$-spikes. In fact, the ratio of Myc tag to FLAG tag is similar to the ratio of hACE2binding to FLAG tag (Fig. 4a, b), indicating that increased ACE2 binding to the $S^{\mathrm{G} 614}$-spikes did not result from its increased affinity to $\mathrm{S}^{\mathrm{G} 614}$ or the open RBD configuration. Instead, these data indicate that a higher number of the S1 domain is present on the surface of $\mathrm{S}^{\mathrm{G} 614}$-expressing cells. To confirm that the monomeric interaction between hACE2 and S protein is not affected by the D614G mutation, we performed surface plasmon resonance assays. Soluble monomeric hACE2-NN bound with a nearly identical affinity to $\mathrm{S}^{\mathrm{G} 614}-\mathrm{Fc}$ and $\mathrm{S} 1^{\mathrm{D} 614}$-Fc immobilized on a chip (Fig. 4c), a result that is expected because the residue 614 is outside of the RBD. Together, these results indicate that increased ACE2 binding to the cell surface-expressed S ${ }^{\mathrm{G} 614}$ spikes is due to decreased S1 shedding.

Finally, we assessed whether differential amount of functional S protein on the virion could influence neutralization sensitivity of the virus. Fig. 4d shows that $\mathrm{PV}^{\mathrm{D} 614}, \mathrm{PV}^{\mathrm{G} 614}$, and $\mathrm{PV}^{\mathrm{FKO}}$ are similarly susceptible to neutralizing plasma derived from convalescent patients, indicating that antibody-mediated control of viruses carrying $\mathrm{S}^{\mathrm{D} 614}$ and $\mathrm{S}^{\mathrm{G} 614}$ would be similar, and that the vaccines based on the D614 genotype will still be effective against the virus carrying the $\mathrm{D} 614 \mathrm{G}$ mutation.

\section{Discussion}

Our data make clear that there are consistent differences in the efficiency with which $S^{D 614}$ and $S^{G 614}$ are incorporated into PVs and VLPs. However, the physical basis for these differences remains undefined. The different ratios of S1:S2 suggest that association between these two domains is weaker in $S^{D 614}$. The apparent instability of $S^{D 614}$ could impact the integrity of the local membrane, appropriate trafficking of the $S$ protein, or appropriate post-translational modification of the S-protein cytoplasmic tail, which may in turn impact S-protein incorporation into the virion. Our data also make clear that both a functional proprotein convertase and a functional furin-cleavage motif are necessary to observe differences between $S^{D 614}$ and $\mathrm{S}^{\mathrm{G} 614}$. 

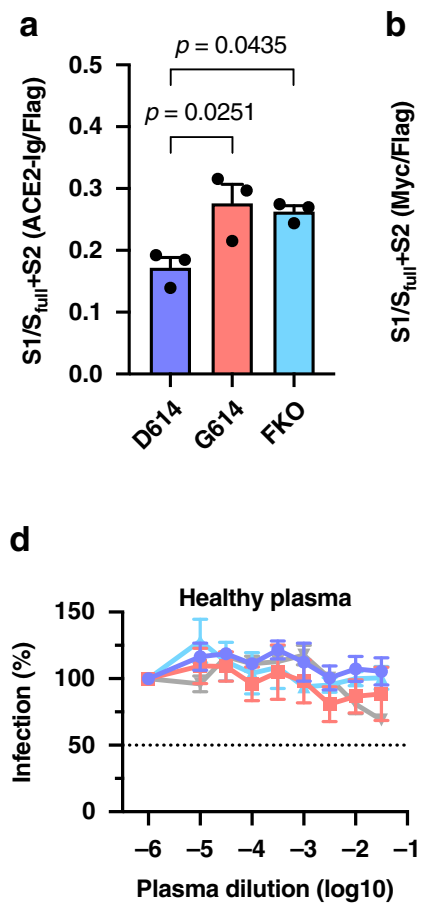
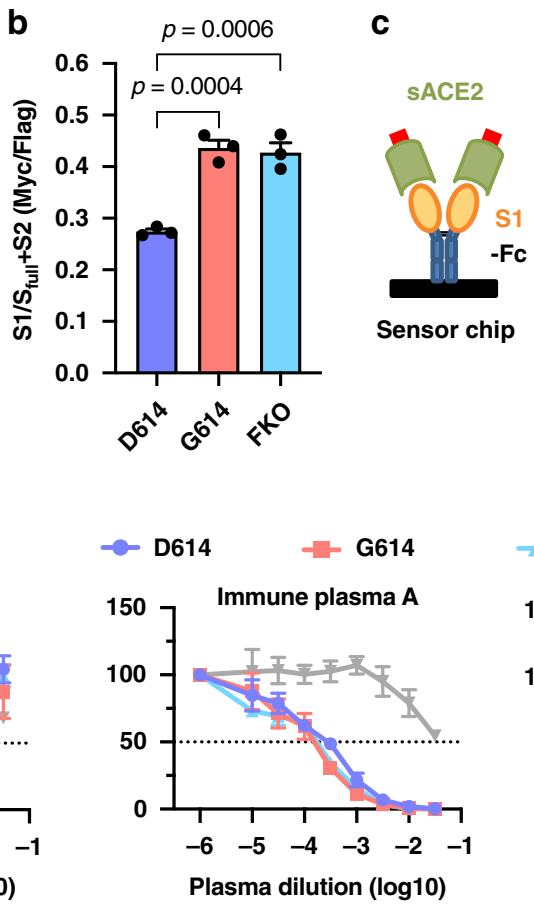
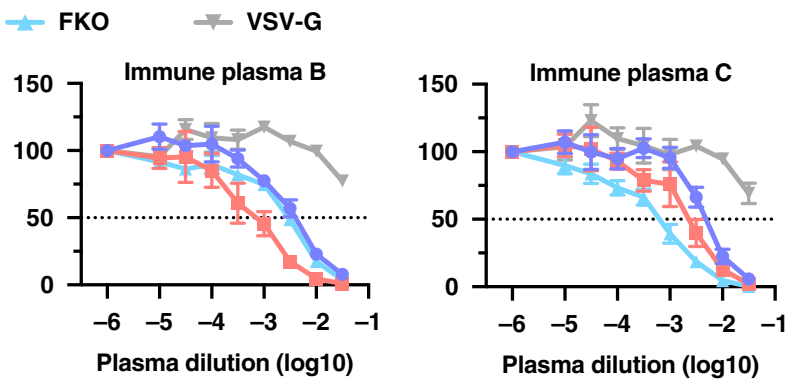

Fig. 4 The D614G mutation neither increases S protein affinity for ACE2 nor makes PV more resistant to neutralization. a The S protein containing Cterminal FLAG tag is transfected into HEK293T cells and assessed for hACE2-NN-lg binding. Total S protein was measured by detecting the FLAG tag in the permeabilized cells. The ratio of hACE2-NN-Ig binding to FLAG-tag staining is shown. $\mathbf{b}$ Experiments similar to those in $\mathbf{a}$ except that the $\mathrm{S}$ protein contains $\mathrm{N}-$ Myc and C-FLAG tags, and S1 level was assessed using an anti-Myc antibody. The data in $\mathbf{a}, \mathbf{b}$ before normalization are presented in Supplementary Fig. 9a, b. Mean \pm SEM of $n=3$ independent experiments are shown. The $p$ values by one-way ANOVA and Sidak multiple comparisons test are indicated. $\mathbf{c}$ Surface plasmon resonance assay design (left) and sensorgrams (middle and right). S1-Fc was immobilized and monomeric hACE2-NN-Ctag40 was injected at 500, $250,125,62.5$, and $31.25 \mathrm{nM}$. Colored lines are the experimental traces and black lines are the best global fits (1:1 Langmuir binding model) used to calculate the association $\left(k_{\text {on }}\right)$ and dissociation $\left(k_{\text {off }}\right)$ rate constants. Representative sensorgrams of three independent experiments with nearly identical results are shown. Supplementary Fig. $9 \mathrm{c}$ shows the proteins used in these assays and Fig. $9 \mathrm{~d}$ presents $k_{\mathrm{on}}, k_{\mathrm{off}}$ and $K_{D}$ values derived from $n=3$ independent experiments. d MLV PVs pseudotyped with the indicated S protein (C-term FLAG) or VSV G protein were preincubated without (presented at $x=-6$ ) or with serially diluted convalescent or control plasmas. hACE2-293T cells were incubated with these preincubated mixes and analyzed $24 \mathrm{~h}$ later by measuring luciferase activity. Mean \pm SEM of $n=3$ independent experiments are presented. FKO furin-cleavage knockout mutant.

Although evidence is still accumulating, the increasing predominance of D614G in humans raises the possibility that viruses with this mutation have a fitness advantage, perhaps allowing more efficient person-to-person transmission. Our virological data are consistent with, but do not themselves demonstrate, this hypothesis. Interestingly, this mutation does not appear to significantly impact disease severity ${ }^{22,27}$. It is possible that higher levels of functional S protein observed with PVG614 increase the chance of host-to-host transmission, but other factors limit the rate and efficiency of intra-host replication. Alternatively, the loss of virion-associated $S$ proteins observed with $S^{\mathrm{D} 614}$ may be compensated by greater fusion efficiency with the destabilized $\mathrm{S}$ protein when the next target cell is adjacent in a host tissue. It is also possible that our ability to detect the emergence of new mutations at this stage of the pandemic is simply greater than our ability to detect modest differences in pathogenesis. The strong phenotypic difference we observe here between D614 and G614 suggests that more studies on the impact of the D614G mutation on the course of disease may be warranted.

Finally, our data raise interesting questions about the natural history of SARS-CoV-2 as it moved presumably from horseshoe bats to humans. At some point in this process, the virus acquired a furin-cleavage site, allowing its S1/S2 boundary to be cleaved in virus-producing cells. In contrast, the S1/S2 boundary of SARS$\mathrm{CoV}-1$, and indeed all SARS-like viruses isolated from bats, civet cats, and pangolins lack this polybasic site and are instead cleaved by TMPRSS2 or endosomal cathepsins in the target cells ${ }^{13-20}$. As we have shown in this study, there are two mechanisms with which SARS-CoV-2 could have enhanced its infectivity, namely, through D614G mutation or elimination of furin-cleavage site with an optimized replacement (Supplementary Fig. 2). However, as the virus transmits in humans, it has clearly retained the furin site while compensating for this site with D614G mutation. Therefore, the furin-cleavage site itself appears to confer a yet-tobe-determined fitness advantage.

In summary, an S-protein mutation, D614G, that appears to promote SARS-CoV-2 transmission in humans also enhances functional S-protein incorporation into SARS-CoV-2 VLP and retroviral PV and increases PV infectivity. Further studies will be necessary to determine the impact of this change on the nature and severity of COVID-19.

\section{Methods}

SARS-CoV-2 S protein sequences analysis. To track the D614G variation among SARS-CoV-2 isolates, $S$ protein sequences were downloaded from GenBank by searching for "SARS-CoV-2 spike" and by filtering the result based on sequence length "1272-1273" to retrieve full-length S protein. To separate retrieved sequences by the month, the result was further filtered by release date. Genotype frequency at residue 614 was calculated using $\mathrm{R}$ version 3.6.0 ( $\mathrm{R}$ Foundation for Statistical Computing) with the Biostrings package version 2.52. Logo plots of D614G variation were generated by WebLogo version 3 after sequence alignment. Total number of sequences analyzed for each month is 17 sequences in January, 33 in February, 293 in March, 1511 in April, and 2544 in May.

MLV PV production. PV production from HEK293T cells: Cells at $\sim 60 \%$ confluence in T75 flasks were transfected by the calcium-phosphate method with $24 \mu \mathrm{g}$ 
Table 1 Plasmids used in the study.

\begin{tabular}{lll} 
Protein & Modification & Note \\
\hline hACE2 & N-terminal Myc and C-term c9 tag & mouseACE2 signal seq + Myc + hACE2 residues 20-805 (NM_021804) \\
& (TETSQVAPA) & \\
hTMPRSS2 & C-terminal FLAG & Full-length (NP_005647.2) \\
hFurin & C-term c9 tag & Full-length (BC012181.1) \\
S1D614-Fc & C-terminal Fc & Residues 1-682; codon-optimized (YP_009724390) \\
S1G614-Fc & C-terminal Fc & Residues 1-682; codon-optimized; D614G mutation \\
hACE2-NN-lg & C-terminal Fc & IGH3 signal seq + hACE2 residues 20-615 (NM_021804) with H374N and \\
& C-terminal Ctag (-EPEA) & H378N mutations \\
hACE2-NN-Ctag & IGH3 signal seq + hACE2 residues 20-615 (NM_021804) with H374N/ \\
MLV gag/pol & None & H378N mutations \\
pQCXIX-eGFP & None & Full length MLV gag and pol \\
pQCXIX-FLuc & None & Full-length eGFP in retroviral vector pQCXIX \\
S protein (D614, G614, or FKO) & C-terminal FLAG & Full-length firefly luciferase in pQCXIX \\
& N-/C-term FLAG & Full-length; codon-optimized (YP_009724390) \\
M protein & No tag & Full-length; codon-optimized; its own signal sequence \\
N protein & None & Full-length; codon-optimized \\
E protein & None & Full-length; codon-optimized (YP_009724393) \\
& None & Full-length; codon-optimized (YP_009724397) \\
& Full-length; codon-optimized (YP_009724392)
\end{tabular}

of total DNA at a ratio of 5:5:1 by mass of the retroviral vector pQCXIX encoding enhanced green fluorescence protein (eGFP) or firefly luciferase (FLuc), a plasmid expressing MLV gag and pol proteins, and a plasmid expressing the $S$ protein of SARS-CoV-2. SARS-CoV-2 $\mathrm{S}$ protein gene was codon-optimized and synthesized by Integrated DNA Technologies based on the protein sequence (GenBank YP_009724390), and cloned in PCAGGS vector. The S protein gene was fused or not to the FLAG tag sequence either at its C-terminus or at both the N- and Ctermini, as indicated in each experiment. More information on the plasmids used in the study is provided in Table 1. Transfected cells were washed at $6 \mathrm{~h}$ post transfection (hpt) and replenished with $10 \mathrm{ml}$ DMEM supplemented with $10 \%$ FBS. PV-containing culture supernatants were collected at $43 \mathrm{hpt}$, cleared through 0.45 $\mu \mathrm{m}$ filters, and either purified or aliquoted and frozen at $-80^{\circ} \mathrm{C}$ immediately.

PV production from NCI-H1299 cells: NCI-H1299 cell line was kindly provided by Joseph Kissil (The Scripps Research Institute, Jupiter, FL, USA), authenticated by ATCC Cell Line Authentication Service, and cells were maintained in RPMI supplemented with $10 \%$ FBS. Cells at $~ 90 \%$ confluence in T75 flasks were transfected with total $24 \mu \mathrm{g}$ DNA using FuGENE (Promega) at a ratio of 1:3 of DNA:FuGENE. Because of low furin activity in these cells, $2 \mu \mathrm{g}$ furin expression plasmid (Table 1) was cotransfected. The remaining $22 \mu \mathrm{g}$ DNA was split at a 5:5:1 ratio among retroviral vector pQCXIX encoding eGFP, a plasmid expressing MLV gag and pol proteins, and a plasmid expressing the $\mathrm{S}$ protein fused to $\mathrm{N}$ - and $\mathrm{C}-$ terminal FLAG tag. $S^{\mathrm{D} 614}, \mathrm{~S}^{\mathrm{G} 614}$, and $\mathrm{S}^{\mathrm{FKO}}$ (Supplementary Fig. 2) were used. Cells were split by trypsinization into two T75 flasks at $6 \mathrm{hpt}$, and replenished with $10 \mathrm{ml}$ RPMI per flask supplemented with $10 \%$ FBS. Culture supernatants containing PVs were collected at $48 \mathrm{hpt}$, cleared of cell debris through centrifugation at $3000 \times g$ for $10 \mathrm{~min}$, and subjected to purification.

PV isolation from intracellular compartments: Transfected cells on a T75 flask were collected, washed once with PBS, and resuspended in $2 \mathrm{ml}$ PBS containing protease inhibitor (Thermo Scientific, A32955). Intracellular PVs were released from three cycles of freezing and thawing, and cell debris was removed by centrifugation for $10 \mathrm{~min}$ at $3000 \times g$. Supernatants were cleared by $0.45 \mu \mathrm{m}$ filtration, the volume was increased to $9 \mathrm{ml}$ with PBS, and subjected to purification.

PV purification. Here, $9 \mathrm{ml}$ of cleared culture supernatants containing PVs were loaded onto $2 \mathrm{ml}$ of $20 \%$ sucrose (Signa-Aldrich, S7903) in NT buffer (120 mM $\mathrm{NaCl}, 20 \mathrm{mM}$ Tris, $\mathrm{pH} 8.0$ ) and centrifuged at $30,000 \mathrm{rpm}$ in the SW41 rotor $(154,000 \times g)$ for $2 \mathrm{~h}$ at $10^{\circ} \mathrm{C}^{36}$. The pellets of PVs derived from HEK293T cells were resuspended in 30-50 $\mathrm{ll}$ NT buffer with gentle shaking on ice and either immediately used or aliquoted and frozen at $-80^{\circ} \mathrm{C}$. The pellets of PVs derived from NCI-H1299 cells were difficult to resuspend, likely because of mucin secreted by lung epithelial cells, and thus resuspended in $30 \mu \mathrm{l}$ NT buffer containing $0.5 \%$ SDS with gentle shaking at room temperature, avoiding bubbling, and used only in WB analyses.

PV quantification. PVs were quantified by RT-qPCR, using primers and a probe that target the CMV promoter. Culture supernatants containing PVs were treated with $100 \mu \mathrm{g} / \mathrm{ml}$ RNase A for $1 \mathrm{~h}$ at $37^{\circ} \mathrm{C}$ to degrade RNAs that are not packaged inside the virion, and RNA was extracted with Trizol and GlycoBlue coprecipitant and digested for $30 \mathrm{~min}$ at $37^{\circ} \mathrm{C}$ with DNase I at $1 \mathrm{IU}$ per $1 \mu \mathrm{g}$ extracted RNA. DNase I was inactivated by incubating for $10 \mathrm{~min}$ at $65^{\circ} \mathrm{C}$ with EDTA added to final $5 \mathrm{mM}$. DNase-treated RNA was reverse transcribed using the High-Capacity cDNA Reverse Transcription Kit (Applied Biosystems, 4374966). qPCR was performed, using Luna Universal Probe qPCR Master Mix (New England Biolabs, M3004E) with the known quantity of PQCXIX vector to generate standard curves and data were collected with CFX Manager 3.1 (Bio-Rad). Primers and a probe were synthesized at Integrated DNA Technologies. Sense primer: 5'-TCACGGGG ATTTCCAAGTCTC-3', anti-sense primer: 5'-AATGGGGCGGAGTTGTTACG AC-3', probe: 5'-FAM-AAACAAACT-(ZEN)-CCCATTGACGTCA-IBFQ-3'.

PV entry assay in HEK293T cells. PV entry assays in HEK293T cells were performed by spinoculating PVs $\left(5 \times 10^{8}\right.$ genome copy/well $)$ onto the Mock-293T, hACE2-293T, or hACE2-293T cells transfected to express hTMPRSS2 seeded on 48-well plates one day before. Spinoculation was carried out at $2100 \times g$ for $30 \mathrm{~min}$ at $25^{\circ} \mathrm{C}$. Spinoculated plates were incubated for $2 \mathrm{~h}$ in a $\mathrm{CO}_{2}$ incubator and medium was replaced with DMEM containing $10 \%$ FBS. Entry levels in GFP expression were measured $24 \mathrm{~h}$ later in the viable cell population gated in pseudocolor plots by Accuri flow cytometer using C6 software 1.0.264 (BD) and ForeCyt 6.2R3 (IntelliCyt). An example of gating strategy is shown in Supplementary Fig. 10. Entry levels in luciferase activity was assessed using the Luc-Pair Firefly Luciferase HS Assay Kit (GeneCopoeia, LF009) and data were collected with SoftMax Pro 6.3 (Molecular Devices).

ACE2 expression and PV entry assay in lung epithelial cell lines. Cells: Calu-3 was recently purchased from ATCC and grown in EMEM supplemented with 10\% FBS. NCI-H1299, NCI-H1975 were kindly provided by Joseph Kissil (The Scripps Research Institute, Jupiter, FL, USA) and maintained in RPMI supplemented with 10\% FBS. NCI-H1299 and NCI-H1975 were authenticated using Short Tandem Repeat analysis through ATCC Cell Line Authentication Service. Cells were grown to approximately $70 \%$ confluence before splitting by trypsinization.

Assessment of ACE2 expression in lung epithelial cell lines: Calu-3, NCIH1299, and NCI-H1975 cells on 6-well plates were detached by scraping in $0.5 \mathrm{ml}$ of PBS containing $5 \mathrm{mM}$ EDTA. hACE2-293T cells were used as a positive control. For hACE2-transduced Calu-3 and NCI-H1975 cells, staining was performed 2 days post transduction. Approximately $0.5 \times 10^{6}$ cells per well in V-bottom 96well plates were incubated on ice for $45 \mathrm{~min}$ with $3 \mu \mathrm{g} / \mathrm{ml}$ anti-hACE2 antibody (R\&D, MAB9332) in PBS containing 2\% goat serum (Gibco, 16210064), followed by goat-anti-mouse IgG conjugated with phycoerythrin (Jackson

ImmunoResearch, 115-116-146) diluted at 1:500 in PBS containing 2\% goat serum. Cells were washed twice with PBS containing $2 \%$ goat serum and once with PBS, fixed with $2 \%$ paraformaldehyde in PBS, and viable cell population gated in pseudocolor plots was analyzed by Accuri flow cytometer using C6 software 1.0.264 (BD) and ForeCyt 6.2R3 (IntelliCyt)

PV entry assay: NCI-H1975 cells were transduced to express hACE2 and seeded next day on 48 -well plates to achieve $~ 30 \%$ at the time of an entry assay. Two days post transduction, cells were spinoculated with PVs $\left(5 \times 10^{8}\right.$ genome copy/well) at $2100 \times g$ for $30 \mathrm{~min}$ at $25^{\circ} \mathrm{C}$. Spinoculated plates were incubated for $2.5 \mathrm{~h}$ in a $\mathrm{CO}_{2}$ incubator, and medium was replaced. Entry levels were assessed $48 \mathrm{~h}$ post infection by measuring GFP expression in the viable cell population gated in pseudocolor plots by Accuri flow cytometer using C6 software 1.0.264 (BD) and ForeCyt 6.2R3 (IntelliCyt).

Analysis of S1 shedding and S protein density of MLV PV. For the analyses of S1 shedding and S-protein density on the virion, purified PVs were analyzed by SDS-PAGE and WB analyses. Approximately $1 / 10$ of purified PVs or VLPs derived from one T75 flask of transfected HEK293T cells or all of purified PVs from two T75 flasks of NCI-H1299 cells (see PV production) were analyzed in each WB analysis.

PV proteins were separated on the 4-12\% Bis-Tris gel (Life Technologies, NW041122), transferred to the PVDF membrane, and blotted with $1 \mu \mathrm{g} / \mathrm{ml}$ anti-FLAG M2 antibody (Sigma-Aldrich, F1804) to detect the S-protein bands or 
$1 \mu \mathrm{g} / \mathrm{ml}$ anti-p30 MLV gag antibody (Abcam, ab130757) to detect $\mathrm{p} 30$ bands as an PV quantity control. Bands were visualized with 1:10,000 diluted goat-anti-mouse IgG-HRP polyclonal antibody (Jackson Immuno Laboratory, 115-036-062) and SuperSignal West Atto Ultimate Sensitivity Substrate (Thermo Scientific, A38555). Band intensities were measured using Image Lab software (Bio-Rad). To increase the accuracy of this measurement, the same blots were analyzed several times at different exposures. Although $\mathrm{PV}^{\mathrm{D} 614}$ and $\mathrm{PV}^{\mathrm{G} 614}$ containing the same amount of S2 domain and $\mathrm{p} 30$ gag would have been useful to confirm differential S1 shedding by these PVs, generating such PVs is technically impossible, owing to the built-in property of SG614 to incorporate into the virion with much higher efficiency than does $S^{\mathrm{D} 614}$

For silver staining, all of the purified PVs from one T75 flask of HEK293T cells were separated by the $4-12 \%$ Bis-Tris gel and stained with Silver Stain Plus kit (Bio-Rad, 1610449). For the PVs produced with the S protein lacking a tag, the S1 and S2 bands were detected using convalescent plasma at 1:250 dilution followed by $10 \mathrm{ng} / \mathrm{ml}$ mouse-anti-human IgG antibody conjugated with polymerized HRP (Fitzgerald, 61R-I166AHRP40) and SuperSignal West Atto Ultimate Sensitivity Substrate.

SARS-CoV-2 VLP production and S-protein analysis. SARS-CoV-2 VLPs were produced by transfecting HEK293T cells at $\sim 60 \%$ confluence in T75 flasks with 25 $\mu \mathrm{g}$ total DNA using the calcium phosphate method. The plasmids expressing SARS-CoV-2 M, N, E, and S proteins were transfected at a ratio of 1:5:5:1. The codon-optimized M, N, and E protein genes were synthesized based on the GenBank protein sequences, YP_009724393, YP_009724397, and YP_009724392, respectively. The S proteins with the FLAG tag at both the $\mathrm{N}$ - and C-termini were used. Transfected cells were washed at $6 \mathrm{hpt}$ and VLPs were harvested $43 \mathrm{hpt}$ from the culture supernatants, cleared by $0.45 \mu \mathrm{m}$ filtration, and purified by pelleting through a $20 \%$ sucrose layer as were MLV PVs. VLP pellets were resuspended in $30 \mu \mathrm{l}$ of NT buffer, and the entire amount derived from one T75 flask was analyzed by SDS-PAGE and WB analysis. The S-protein bands were detected using the anti-FLAG M2 antibody, and the N-protein bands were detected using pooled convalescent plasma at a 1:500 dilution. Bands were visualized with $10 \mathrm{ng} / \mathrm{ml}$ mouse-anti-human IgG antibody conjugated with polymerized HRP (Fitzgerald, 61R-I166AHRP40) and SuperSignal West Atto Ultimate Sensitivity Substrate.

\section{Cell-surface expression and analysis of the $\mathbf{S}$ protein. HEK293T cells,} approximately $80 \%$ confluent in 6-well plates were transfected with $8 \mu$ PEI 40,000 (Polysciences) and $2 \mu \mathrm{g}$ plasmid expressing the indicated $\mathrm{S}$ protein variant. For ACE2-NN-Ig binding experiments, the $S$ protein constructs with the FLAG tag at the C-terminus were used. NN denotes the mutations of two histidines to asparagines at the positions 374 and 378, which inactivate ACE2 enzyme activity but do not affect S-protein binding ${ }^{39}$. To measure $S 1$ and total S protein, the $S$ proteins with $\mathrm{N}$-terminal Myc tag and C-terminal FLAG tag were used. All tags were fused with a two-glycine (GG) linker. To measure ACE2-binding ability or the level of the S1 domain present on the cell surface, cells were detached two days post transfection with Accutase (Stemcell Technologies Inc.) and approximately $5 \times 10^{5}$ cells were incubated on ice in $100 \mu \mathrm{l}$ of either $1 \mu \mathrm{g} / \mathrm{ml}$ purified hACE2-NN-Ig followed by 1:200 diluted anti-hIgG-FITC (Jackson ImmunoResearch, 109-096097) or $3 \mu \mathrm{g} / \mathrm{ml}$ anti-Myc antibody (clone 9E10, National Cell Culture Center, Minneapolis, MN) followed by 1:200 diluted anti-mIgG-APC (Jackson ImmunoResearch, 115-136-072), respectively. hACE2-NN-Ig was purified using Protein A-Sepharose CL-4B (GE Healthcare). To measure total level of the $S$ protein, cells were permeabilized with $0.5 \%$ Triton X-100 (Sigma-Aldrich) in PBS for $10 \mathrm{~min}$ at room temperature and incubated with $1 \mu \mathrm{g} / \mathrm{ml}$ anti-FLAG M2 antibody (SigmaAldrich, F1804) followed by 1:200 diluted anti-mIgG-APC (Jackson ImmunoResearch, 115-136-072)

Surface Plasmon Resonance (SPR) analysis. The proteins used in SPR analyses were produced from transient transfection of Expi293 (Thermo-Fisher) using FectoPRO (Polyplus) with plasmids encoding the Fc-fusion forms of SARS-CoV-2 S1 domain (S1-Fc, residues 1-682) or hACE2-NN (residues 20-615) with a Cterminal Ctag $(-\mathrm{EPEA})^{40}$. After 5 days in shaker culture, media were collected and cleared of debris by low-speed centrifugation and $0.45-\mu \mathrm{m}$ filtration. S1-Fc was purified using MabSelect Sure (GE Lifesciences) and hACE2-NN-Ctag using CaptureSelect C-tagXL (Thermo-Fisher) columns according to the manufacturer's instruction. The buffer of eluates was exchanged with PBS and concentrated using Amicon ultra filtration devices (Millipore Sigma), and proteins were stored at $4{ }^{\circ} \mathrm{C}$ and used within a few days.

SPR measurements were performed on a Biacore X100 instrument at $25^{\circ} \mathrm{C}$ using $1 \mathrm{x}$ HBS-EP + (Cytiva) as running buffer. A mouse anti-human $\operatorname{IgG~}_{\mathrm{H}} 2$ monoclonal antibody (Cytiva) was immobilized to a density of $\sim 3800$ response units (RU) on a CM5 sensor chip via standard NHS/EDC coupling methods (Cytiva). Subsequently, S1 ${ }^{\mathrm{D} 614}-\mathrm{Fc}$ or S1 ${ }^{\mathrm{G} 614}-\mathrm{Fc}$, each at $10 \mu \mathrm{g} / \mathrm{mL}$, was captured to $\sim 680 \mathrm{RU}$ on the active flow cell. A concentration series with two-fold dilutions (500-31.25 nM) of monomeric hACE2-NN-Ctag were injected using a multi-cycle method. The lowest concentration $(31.25 \mathrm{nM})$ was repeated to confirm regeneration of the sensor chip. Biacore X100 Control Software 2.0.1 (Cytiva) was used to collect data and Biacore X100 Evaluation Software 2.0.1 (Cytiva) to analyze data. Calculation of association $\left(k_{\text {on }}\right)$ and dissociation $\left(k_{\text {off }}\right)$ rate constants was based on a 1:1 Langmuir binding model. All fitting data revealed a $\mathrm{Chi}^{2}$ value of $<1$. The equilibrium dissociation constant $\left(K_{D}\right)$ was calculated from $k_{\text {off }} / k_{\text {on. }}$.

Neutralization assay with human immune plasma. Deidentified blood samples were obtained by the Allergy, Asthma and Immunology Specialists of South Florida, LLC for COVID-19 serotyping, and exempt (IRB-20-7580) from human subject research CFR 45.101(b)(4). MLV PVs encoding firefly luciferase and pseudotyped with the indicated SARS-CoV-2 S protein were preincubated for $1 \mathrm{~h}$ at $37^{\circ} \mathrm{C}$ with or without convalescent or healthy plasma serially diluted in DMEM containing 10\% FBS. Preincubated samples were added to Mock- and hACE2-293T cells on 96-well plates and entry levels were assessed $24 \mathrm{~h}$ later by measuring luciferase activity using the Luc-Pair Firefly Luciferase HS Assay Kit (GeneCopoeia, LF009) and data collected with SoftMax Pro 6.3 (Molecular Devices).

Statistical analysis. All appropriate data were analyzed with GraphPad Prism version 7 (GraphPad Software Inc.). All hypothesis tests were performed as twotailed tests. Specific statistical analysis methods are described in the figure legends where results are presented. Values were considered statistically significant for $p$ values below 0.05 . Exact $p$ values are provided in appropriate figures.

\section{Reagent availability}

Plasmids and cell lines used in this study are available from the corresponding author upon request.

Reporting summary. Further information on research design is available in the Nature Research Reporting Summary linked to this article.

\section{Data availability}

Source Data are provided with this paper and all relevant data are available from the authors. The data used to generate the Cryo-EM images in Fig. la are available from PBD entry 6VXX (http://www.rcsb.org/structure/6vxx). Sequences used to generate logo plots in Fig. $1 \mathrm{~b}$ are available from GenBank (https://www.ncbi.nlm.nih.gov/protein) by searching for "SARS-CoV-2 spike" and filtering the result by the length of the protein (1272-1273). To separate the result by the month, retrieved sequences can be further filtered by the release date. Source data are provided with this paper.

Received: 23 September 2020; Accepted: 27 October 2020; Published online: 26 November 2020

\section{References}

1. Li, W., Choe, H. \& Farzan, M. Insights from the association of SARS-CoV S-protein with its receptor, ACE2. Adv. Exp. Med. Biol. 581, 209-218 (2006).

2. Fehr, A. R. \& Perlman, S. Coronaviruses: an overview of their replication and pathogenesis. Methods Mol. Biol. 1282, 1-23 (2015).

3. Shang, J. et al. Structural basis of receptor recognition by SARS-CoV-2. Nature 581, 221-224 (2020).

4. Zhou, P. et al. A pneumonia outbreak associated with a new coronavirus of probable bat origin. Nature 579, 270-273 (2020).

5. Walls, A. C. et al. Structure, function, and antigenicity of the SARS-CoV-2 spike glycoprotein. Cell, 181, P281-292.E6 (2020).

6. Lan, J. et al. Structure of the SARS-CoV-2 spike receptor-binding domain bound to the ACE2 receptor. Nature 581, 215-220 (2020).

7. Wang, Q. et al. Structural and functional basis of SARS-CoV-2 entry by using human ACE2. Cell 181, 894-904 (2020).

8. $\mathrm{Li}, \mathrm{W}$. et al. Angiotensin-converting enzyme 2 is a functional receptor for the SARS coronavirus. Nature 426, 450-454 (2003)

9. $\mathrm{Li}, \mathrm{W}$. et al. Receptor and viral determinants of SARS-coronavirus adaptation to human ACE2. EMBO J. 24, 1634-1643 (2005).

10. Wong, S. K., Li, W., Moore, M. J., Choe, H. \& Farzan, M. A 193-amino acid fragment of the SARS coronavirus S protein efficiently binds angiotensinconverting enzyme 2. J. Biol. Chem. 279, 3197-3201 (2004).

11. Li, F., Li, W., Farzan, M. \& Harrison, S. C. Structure of SARS coronavirus spike receptor-binding domain complexed with receptor. Science 309, 1864-1868 (2005)

12. Shang, J. et al. Cell entry mechanisms of SARS-CoV-2. Proc. Natl. Acad. Sci. USA 117, 11727-11734 (2020).

13. Glowacka, I. et al. Evidence that TMPRSS2 activates the severe acute respiratory syndrome coronavirus spike protein for membrane fusion and reduces viral control by the humoral immune response. J. Virol. 85, 4122-4134 (2011) 
14. Huang, I. C. et al. SARS coronavirus, but not human coronavirus NL63, utilizes cathepsin L to infect ACE2-expressing cells. J. Biol. Chem. 281, 3198-3203 (2006).

15. Matsuyama, S. et al. Efficient activation of the severe acute respiratory syndrome coronavirus spike protein by the transmembrane protease TMPRSS2. J. Virol. 84, 12658-12664 (2010).

16. Millet, J. K. \& Whittaker, G. R. Host cell proteases: critical determinants of coronavirus tropism and pathogenesis. Virus Res. 202, 120-134 (2015).

17. Shulla, A. et al. A transmembrane serine protease is linked to the severe acute respiratory syndrome coronavirus receptor and activates virus entry. J. Virol. 85, 873-882 (2011).

18. Simmons, G. et al. Inhibitors of cathepsin L prevent severe acute respiratory syndrome coronavirus entry. Proc. Natl. Acad. Sci. USA 102, 11876-11881 (2005).

19. Belouzard, S., Chu, V. C. \& Whittaker, G. R. Activation of the SARS coronavirus spike protein via sequential proteolytic cleavage at two distinct sites. Proc. Natl Acad. Sci. USA 106, 5871-5876 (2009).

20. Hoffmann, M. et al. SARS-CoV-2 cell entry depends on ACE2 and TMPRSS2 and is blocked by a clinically proven protease inhibitor. Cell, 181, 271-280.e8. (2020).

21. Júnior, I. J. M., Polveiro, R. C., Souza, G. M., Inserra, D. \& Bortolin, F. T. S., Lima A. T. M. The global population of SARS-CoV-2 is composed of six major subtypes. bioRxiv, https://doi.org/10.1101/2020.04.14.040782 (2020).

22. Korber, B. et al. Tracking changes in SARS-CoV-2 Spike: evidence that D614G increases infectivity of the COVID-19 virus. Cell182, 812-827 (2020).

23. Laha, S. et al. Characterizations of SARS-CoV-2 mutational profile, spike protein stability and viral transmission. Infect. Genet. Evol. 85, 104445. https:// doi.org/10.1016/j.meegid.2020.104445 (2020).

24. Laamarti, M. et al. Large scale genomic analysis of 3067 SARS-CoV-2 genomes reveals a clonal geo-distribution and a rich genetic variations of hotspots mutations. bioRxiv, https://doi.org/10.1101/2020.05.03.074567.

25. Lokman, S. M. et al. Exploring the genomic and proteomic variations of SARS-CoV-2 spike glycoprotein: a computational biology approach. bioRxiv, https://doi.org/10.1101/2020.04.07.030924 (2020).

26. Comandatore, F. et al. Insurgence and worldwide diffusion of genomic variants in SARS-CoV-2 genomes. bioRxiv, https://doi.org/10.1101/ 2020.04.30.071027 (2020).

27. Zhang, L. et al. Genome-wide variations of SARS-CoV-2 infer evolution relationship and transmission route. bioRxiv, https://doi.org/10.1101/ 2020.04.27.20081349 (2020).

28. van Dorp, L. et al. No evidence for increased transmissibility from recurrent mutations in SARS-CoV-2. bioRxiv, https://doi.org/10.1101/ 2020.05.21.108506 (2020).

29. Bhowmik, D., Pal, S., Lahiri, A., Talukdar, A. \& Paul, S. Emergence of multiple variants of SARS-CoV-2 with signature structural changes. bioRxiv, https:// doi.org/10.1101/2020.04.26.062471 (2020).

30. Cortey, M. et al. SARS-CoV-2 amino acid substitutions widely spread in the human population are mainly located in highly conserved segments of the structural proteins. bioRxiv, https://doi.org/10.1101/2020.05.16.099499 (2020).

31. Muller, N.F. et al. Viral genomes reveal patterns of the SARS-CoV-2 outbreak in Washington State. medRxiv https://doi.org/10.1101/2020.09.30.20204230 (2020).

32. Hoffmann, M., Kleine-Weber, H. \& Pohlmann, S. A multibasic cleavage site in the spike protein of SARS-CoV-2 is essential for infection of human lung cells. Mol. Cell 78, 779-784 (2020).

33. Lukassen, S. et al. SARS-CoV-2 receptor ACE2 and TMPRSS2 are primarily expressed in bronchial transient secretory cells. EMBO J. 39, e105114 (2020).

34. Muss, C. et al. Integrated analyses of single-cell atlases reveal age, gender, and smoking status associations with cell type-specific expression of mediators of SARS-CoV-2 viral entry and highlights inflammatory programs in putative target cells. bioRxiv, https://doi.org/10.1101/2020.04.19.049254 (2020).

35. Ziegler, C. G. K. et al. SARS-CoV-2 receptor ACE2 is an interferon-stimulated gene in human airway epithelial cells and is detected in specific cell subsets across tissues. Cell 181, 1016-1035 (2020).
36. Ou, X. et al. Characterization of spike glycoprotein of SARS-CoV-2 on virus entry and its immune cross-reactivity with SARS-CoV. Nat. Commun. 11, 1620 (2020).

37. Huang, Y., Yang, Z. Y., Kong, W. P. \& Nabel, G. J. Generation of synthetic severe acute respiratory syndrome coronavirus pseudoparticles: implications for assembly and vaccine production. J. Virol. 78, 12557-12565 (2004).

38. Wrapp, D. et al. Cryo-EM structure of the 2019-nCoV spike in the prefusion conformation. Science 367, 1260-1263 (2020).

39. Moore, M. J. et al. Retroviruses pseudotyped with the severe acute respiratory syndrome coronavirus spike protein efficiently infect cells expressing angiotensin-converting enzyme 2. J. Virol. 78, 10628-10635 (2004).

40. De Genst, E. J. et al. Structure and properties of a complex of alpha-synuclein and a single-domain camelid antibody. J. Mol. Biol. 402, 326-343 (2010).

\section{Acknowledgements}

This work is supported by an administrative supplement to NIH R01 AI129868 for coronavirus research (awarded to M.F. and H.C.), and by NSF-RAPID award MCB2033939, using funds provided by the 2020 CARES Act (awarded to T.I.).

\section{Author contributions}

H.C. and M.F. conceived of and supervised the study. L.Z., C.B.J., H.M., C.R., M.F., and H.C. designed experiments. L.Z., C.B.J., H.M., A.O., H.P., B.D.Q., A.P., A.V., and M.S.S performed experiments. L.Z., C.B.J., H.M., A.O., and H.P. analyzed data. E.S.R., W.L., and T.I. provided essential reagents. M.F. and H.C. wrote the manuscript. All authors reviewed and edited the manuscript. L.Z., C.B.J., and H.M. equally contributed to the study.

\section{Competing interests}

The authors claim no competing interest.

\section{Additional information}

Supplementary information is available for this paper at https://doi.org/10.1038/s41467020-19808-4.

Correspondence and requests for materials should be addressed to M.F. or H.C.

Peer review information Nature Communications thanks the anonymous reviewers for their contribution to the peer review of this work.

Reprints and permission information is available at http://www.nature.com/reprints

Publisher's note Springer Nature remains neutral with regard to jurisdictional claims in published maps and institutional affiliations.

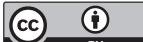

Open Access This article is licensed under a Creative Commons Attribution 4.0 International License, which permits use, sharing, adaptation, distribution and reproduction in any medium or format, as long as you give appropriate credit to the original author(s) and the source, provide a link to the Creative Commons license, and indicate if changes were made. The images or other third party material in this article are included in the article's Creative Commons license, unless indicated otherwise in a credit line to the material. If material is not included in the article's Creative Commons license and your intended use is not permitted by statutory regulation or exceeds the permitted use, you will need to obtain permission directly from the copyright holder. To view a copy of this license, visit http://creativecommons.org/ licenses/by/4.0/.

(C) The Author(s) 2020 